\title{
Gestión del conocimiento sustentable universitario Visión aproximada de experiencias latinoamericanas ${ }^{*}$
}

\author{
Beatriz Carolina Carvajal ${ }^{* *}$
}

Recibido: 7 de marzo de 2014

Evaluado: 18 de abril de 2014

Aceptado: 10 de mayo de 2014

\section{Resumen}

En la investigación que sustenta este artículo, se desarrolló como objetivo: el análisis de experiencias de gestión de conocimiento en universidades latinoamericanas (Venezuela, Argentina, Brasil). Con base en ese análisis, se plantearon lineamientos generales para la gestión del conocimiento sustentable. Se propone el concepto de gestión del conocimiento sustentable como abstracción teórica, que permite la conexión tanto del constructo de co-inspiración (Maturana, 1997a, 1997b) como del criterio de autopoiesis (Maturana y Valera, 1999), con el principio de una mente sustentable (Vieira, 2011). Desde una perspectiva transdisciplinar en la que convergen: la sociología, la biología y la ecología, la problemática de la gestión de conocimiento es vista como una articulación de procesos sociales y ecológicos, que pueden ser comprendidos con la mediación de diversas disciplinas que posibilitarían la gestión del conocimiento a futuro. Los lineamientos generales postulados se fundan en la reflexión teórica y en la interpretación de las entrevistas semiestructuradas a expertos en el área de gestión de conocimiento de las universidades seleccionadas en el muestreo intencional, a saber: a) en Venezuela: Universidad Centroccidental Lisandro Alvarado (UCLA), Universidad del Zulia (LUZ), Universidad Nacional Experimental Rafael María

En este artículo se presentan algunos de los resultados de la investigación: "Gestión del conocimiento sustentable universitario", la cual ha sido financiada por el Consejo de Desarrollo Científico, Tecnológico y Humanístico (CDCHT) de la Universidad Centroccidental Lisandro Alvarado, durante los años 2012 y 2013. Cómo citar este artículo: Carvajal, B. C (2014). Gestión del conocimiento sustentable universitario. Visión aproximada de experiencias latinoamericanas. Hallazgos, 11 (22), pp. 159-181.

** Postdoctora egresada del Programa Multidisciplinario de Formación Continua para Doctores en Ciencias Sociales, Ciencias de la Comunicación, Humanidades y Artes, Universidad Nacional de Córdoba, Argentina, año 2008; Profesora Titular en la Universidad Centroccidental "Lisandro Alvarado" (Venezuela). E-mail: beatrizcarvaja@@ucla.edu.ve, becar777@yahoo.es. Barquisimeto, Estado Lara; Venezuela. 
Baralt (UNERMB), y la Universidad de Carabobo (UC); b) en Argentina: Universidad de Buenos Aires y Universidad de Córdoba, y c) en Brasil: Instituto Federal Sul Rio Grandense y la Universidad Federal de Río de Janeiro (UFRJ). Se concluye que la producción y la gestión del conocimiento académico sustentable han sido importantes inquietudes históricas de los académicos latinoamericanos; se agrega que esos problemas tienen expresiones específicas en los diferentes países. Factores de orden socioeconómico, político y ecológico, tales como pobreza, hambre, desigual distribución del ingreso global, indisponibilidad de recursos médicos y biotecnológicos y daño ecológico, sólo por mencionar algunos, representan una realidad que impulsa a los académicos hacia la búsqueda de respuestas decisivas, planteando la urgencia en la búsqueda de la renovación en la gestión del conocimiento universitario. La propuesta es hacer coexistir, como igualmente importantes y sin subordinación el uno del otro, dos focos de referencia: la organización universitaria como un todo y cada una de las personas que la constituyen; una gestión precedida por la inter- $\mathrm{y}$ transdisciplinariedad del conocimiento, y sostenida en una red conversacional de comprensión, inclusión, y co-inspiración.

Palabras clave: Gestión, conocimiento, sustentabilidad, co-inspiración, autopoiesis y mente sustentable. 


\title{
Management of sustainable academic knowledge Vision Approximate of Latin American Experiences
}

\begin{abstract}
The research objective was analysis of knowledge management experiences in Latin American universities (Venezuela, Argentina, and Brazil). And analysis based on general guidelines that were raised in sustainable knowledge management. We are going to present a paper derivate from that research. The problem is approached from a trans-disciplinary perspective through Sociological, Biological and Ecological theories, in order to look at knowledge management, as the dynamic process that occurs by simultaneous articulation of social and ecological factors. This viewpoint - so mediated by several disciplines - would permit a clearer comprehension of the problem and could make possible a better management of knowledge in the future. Methodology: The author focusses on the concept of sustainable knowledge as a theoretical abstraction, that connects both the construct of coinspiration (Maturana, 1997a, 1997b) and autopoiesis (Maturana \& Valera, 1999), with the principle of a sustainable mind (Vieira, 2011). Theoretical reflections are based on the results obtained from the analyses of semi-structured interviews, that were administered to scholars - who function as management of knowledge experts - at the following selected centers: a) Venezuela; Universidad Centroccidental "Lisandro Alvarado" (UCLA), Universidad del Zulia (LUZ), Universidad Nacional Experimental "Rafael María Baralt" (UNERMB) and the Universidad de Carabobo (UC); b) Argentina; Universidad de Buenos Aires and Universidad de Córdoba; and in c) Brasil; Instituto Federal Sul Rio Grandense and the Universidad Federal de Río de Janeiro (UFRJ). Conclusions: production and management of sustainable academic knowledge have been hefty
\end{abstract}


concerning matters for Latin-American scholars; besides, it has specific expressions within different countries. To worsen the difficulties, functionality of those academic centers is constantly modified by the impact of critical sociologic, political, ecological and economic factors; such as: poverty, hunger, uneven distribution of global income, availability of medical and biotechnological resources and ecological damage; just to mention a few. This reality should prompt academicians to look for responses to such a complex set of problems. Recommendations: To make the University actively function as an entire participant, by means of the incorporation of every one of its human components; so to process the management of knowledge through inter and transdisciplinary approach. And once that commitment is reached, to establish a conversational net of comprehension, inclusion and co-inspiration with the people the Academy is supposed to serve.

Keywords: Management, knowledge, sustainable, co-inspiration, autopoiesis, mind. 


\section{Gestão do conhecimento sustentável universitário}

\section{Vista aproximada de experiências latino-americanas}

\section{Resumo}

A pesquisa subjacente a este artigo foi desenvolvido como objetivo: a análise de experiências de gestão do conhecimento em universidades latino-americanas (Venezuela, Argentina, Brasil). Com base nesta análise, foram planteadas as diretrizes gerais para a gestão do conhecimento sustentável. Propõe-se o conceito de gestão do conhecimento sustentável como uma abstração teórica, que permite a conexão tanto do constructor de co-inspiração (Maturana, 1997a, 1997b) como do critério de autopoiese (Maturana e Valera, 1999), com o princípio da uma mente sustentável (Vieira, 2011). A partir de uma perspectiva transdisciplinar na qual convergem: a sociologia, a biologia e a ecologia, o problema da gestão do conhecimento é visto como uma articulação de processos sociais e ecológicos que podem ser entendidas com a mediação de várias disciplinas que possibilitam a gestão do conhecimento no futuro. Os linhamentos gerais postulados baseam-se na reflexão teórica e na interpretação das entrevistas, semi-estruturadas, para especialistas na área de gestão do conhecimento em universidades selecionadas na amostragem intencional, a saber: a) Venezuela; Universidad Centro-ocidental "Lisandro Alvarado" (UCLA), Universidad del Zulia (LUZ), Universidad National Experimental "Rafael María Baralt" (UNERMB) e a Universidade de Carabobo (UC); b) Argentina; Universidade de Buenos Aires e a Universidade de Córdoba; e c) Brasil; Instituto Federal Sul Rio Grandense e a Universidade Federal do Rio de Janeiro (UFRJ). Cumclue-se então: a produção e a gestão do conhecimento acadêmico sustentável, têm sido importantes preocupações históricas dos académicos latino-americanos; acrescenta-se que 
estes problemas têm expressões específicas nos diversos países. Fatores sócio-econômicos, políticos e ecológicos, tais como a pobreza, a fome, a distribuição desigual da renda global, indisponibilidade de recursos médicos e biotecnológicos e o dano ecológico, só para citar alguns, representam uma realidade que leva os académicos para a procura de respostas decisivas, propondo a urgência na busca da renovação da gestão do conhecimento universitário. A proposta: fazer coexistir como igualmente importantes e sem subordinação de um ao outro, dois marcos de referência: a organização universitaria como um todo e cada uma das pessoas que a constituem; uma gestão precedida pela inter e transdisciplinar do conhecimento, e sostenida numa rede conversacional de compreensão, inclusão e co-inspiração.

Palavras-chave: Gestão, conhecimento, sustentabilidade, co-inspiração, autopoiesis e mente sustentável. 


\section{Presentación}

Partiendo de la premisa de que aun cuando las universidades latinoamericanas han impulsado cambios y, con ello, propuestas que resultan novedosas en la gestión del conocimiento, estas prácticas pueden no estar coherentemente en sintonía con las realidades sociales, económicas, políticas y medioambientales de sus entornos (Carvajal, 2003; 2011). Se considera que la universidad, como organización, está llamada a cambiar y, con ello, a ajustar su gestión del conocimiento, ante un entorno que se caracteriza por las presiones que ejercen los diferentes actores o stakeholers.

En efecto, debe dar respuestas a un contexto político-económico mundial, que demanda réplicas ante problemas como las grandes desigualdades sociales, el deterioro del medioambiente, la urgencia de su preservación prospectiva y las pandemias ya avizoradas por la OMS; estas respuestas se han de producir desde los ámbitos político-humanístico y científico-tecnológico en una gestión de conocimiento sustentable y proactiva.

En otras palabras, los universitarios estamos llamados a asumir sin dilaciones la renovación de las universidades, y acompañar los cambios del entorno y más urgente aún, protagonizarlos. Una vía para movilizar esos cambios desde la universidad es por medio de la gestión del conocimiento, tal y como lo han demostrado las universidades a lo largo de su historia con la producción de conocimientos, que ha sido de utilidad para la sociedad en general y para la generación de nuevas interrogantes en el ámbito científico en particular.
Especialmente, si se observa que las instituciones de educación superior reconocen y lideran su rol potencial en la gestión del conocimiento, por medio de sus instancias de investigación decanatos, institutos, centros, líneas y proyectos de investigación, puede verse que estas instancias deberían estar comprometidas con la generación de conocimiento, como única fuente de ventaja competitiva sostenible para la solución de los problemas más elementales que demandan el contexto social, el económico, el ambiental y el cultural.

Entonces la gestión del conocimiento ha de orientarse hacia la clasificación, almacenamiento y transferencia de los conocimientos a su entorno, de tal manera que estos conocimientos serían compartidos por los actores sociales de la organización universitaria y reescritos de acuerdo a los cambios contextuales, a los cuales la organización tendrá que adaptarse de manera proactiva y pensando en el futuro.

Vista la gestión del conocimiento de este modo proactivo, se incorpora el supuesto de sustentabilidad al de gestión del conocimiento, lo cual da origen a la abstracción teórica en la que se relaciona el constructo de co-inspiración (Maturana, 1997a, Maturana, 1997b), la autopoiesis de los sistemas sociales (Maturana y Varela, 1999) y el principio de mente sustentable (Vieira, 2011). La propuesta teórica que aquí se formula conlleva a la aplicación de un enfoque gestáltico, inter y transdisciplinario en la gestión del conocimiento.

Así, desde una perspectiva transdisciplinar en la que convergen la sociología, la biología y la ecología, la problemática de la gestión de conocimiento es vista como una articulación de procesos sociales y ecológicos, que pueden 
ser interpretados con la mediación de diversas disciplinas que posibilitarían la gestión del conocimiento para las generaciones futuras.

En esta disertación se presentan los resultados de una investigación cuyo propósito fue la realización de un estudio que permitiera la comparación de la gestión del conocimiento en algunas universidades latinoamericanas (Venezuela, Argentina, Brasil), para proponer lineamientos generales que viabilicen la gestión del conocimiento sustentable en instituciones de educación superior.

La ruta metódica seguida en dicha investigación estuvo fundada en: a) la revisión documental, es decir, en la reflexión e interpretación de los diferentes aportes teóricos relacionados con el sujeto-objeto de estudio seleccionado; y b) la interpretación, a la manera hermenéutica, de entrevistas a informantes clave, de las universidades seleccionadas en la muestra no probabilística. El tipo de muestreo fue intencional, toda vez que obedeció a criterios prefijados en la pesquisa ${ }^{1}$, y tuvo como unidad de selección las siguientes universidades: Venezuela: Universidad Centroccidental Lisandro Alvarado (UCLA), Universidad del Zulia (LUZ) y la Universidad Nacional Experimental Rafael María Baralt (UNERMB); Argentina: Universidad de Buenos Aires y Universidad de Córdoba; Brasil: Instituto Federal Sul Rio Grandense y la Universidad Federal de Río de Janeiro (UFRJ).

Los criterios prefijados para la selección de la muestra, en concordancia con los objetivos de investigación (comparar las experiencias significativas en la gestión del conocimiento sustentable en instituciones de educación superior latinoamericanas, y formular lineamientos generales para la gestión del conocimiento sustentable), así también con la certeza de lograr contactar a los informantes clave en cada una de las universidades seleccionadas, las cuales debían cumplir con el criterio de ser universidades públicas.
Se intenta así promover la disertación de cambios en el ámbito de la educación superior, específicamente en lo referente a la sustentabilidad en su gestión del conocimiento. De tal manera contribuir con elementos propositivos con miras a plantear lineamientos generales, que inviten a colocar a las universidades de la región a la vanguardia de los entornos cambiantes.

\section{Discusión TEÓRICA}

La crisis del pensamiento que se viene debatiendo desde mediados del siglo pasado (Habermas, 2004; Moreno, 1995) y finales de los años noventa en América Latina ${ }^{2}$ (Martínez, 2009, Parra, 2008,), tiene variadas aristas, una de las más controvertidas está directamente relacionada con el papel de las universidades en la gestión y producción de conocimiento.

Las universidades públicas latinoamericanas (UPL) asumieron la decadencia de la racionalidad científico técnica, que comenzó a ser una realidad insistentemente discutida entre los académicos latinoamericanos de finales del siglo XX; producir conocimiento desde unas ciencias en crisis o generar nuevos paradigmas era el dilema preponderante en esos años.

Ya iniciada la segunda década del siglo XXI, la situación no parece haber variado mucho (Carvajal y Carvajal, 2010), y aquí ya no se trata únicamente de producir conocimiento, sino de gestionarlo estando alerta a los cambios del entorno, y comprometidos con la

2 La decadencia de la racionalidad científico-técnica comenzó a ser una realidad insistentemente discutida entre los académicos latinoamericanos de finales del siglo XX, en la que se planteó la disyuntiva: producir conocimiento desde unas ciencias en crisis o generar nuevos paradigmas. 
capacidad de cambio del entorno externo y del interno, donde la disciplina, el esfuerzo y el pensamiento son relevantes en la producción de conocimiento.

Así, la disyuntiva en cuanto a producir conocimiento desde unas ciencias en crisis, o generar nuevos paradigmas, ha devenido en el cuestionamiento a la concepción tradicional de la gestión del conocimiento, para puntualizar alternativas más novedosas y flexibles. Se plantea un cambio de paradigma, cuyo fundamento se enraíza en la inter y la transdisciplinariedad como cimiento epistemológico, cuyo fin último es la búsqueda de sentido para el desarrollo humano y social. En este particular resalta la reflexión de Martínez quien señala:

En todos los campos se constata que la mayoría de los problemas no pueden resolverse al nivel en que son planteados, ya que su naturaleza forma como un ribosoma complejo de muy variadas interacciones. Por todo ello, la tarea a realizar no es fácil [...] El mundo en el que hoy vivimos, se caracteriza por sus interconexiones a un nivel amplio y global, en el que los fenómenos físicos biológicos, psicológicos, sociales y ambientales son todos recíprocamente interdependiente. Para describir este mundo de una manera adecuada necesitamos una perspectiva más amplia, holista, sistémica y ecológica. Que no nos pueden ofrecer los conceptos reduccionistas del mundo, ni las diferentes disciplinas aisladas. Y menos aún, con términos, conceptos y un lenguaje calcificado en el tiempo y en el espacio. (Martínez, 2008, p. 2).
Desde esa premisa de Martínez se puede colegir que a las UPL se les presenta hoy día, entre otros desafíos, el de desarrollar su capacidad para adaptarse con agilidad a las demandas y necesidades de su entorno, desde la inter- y la transdisciplinariedad en la gestión del conocimiento, propiciando con esta praxis la sustentabilidad del mismo.

La sustentabilidad del conocimiento es concebida como un desafío para las universidades, a dar respuestas ante las causas de la crisis de recursos, las desigualdades del desarrollo económico, la distribución social de los costos ecológicos y dar cuenta de las nuevas estrategias de aprovechamiento y explotación de los recursos.

Ciertamente, la universidades públicas latinoamericanas tienen la oportunidad de atender a los desafíos que plantea el entorno socio-productivo, generando un conocimiento ya no de forma estereotipada, en el que no es posible el libre pensamiento, el cuestionamiento y la reflexión. Por el contrario, la praxis estaría marcada por una concepción de inter y transdisciplinariedad en la gestión del conocimiento.

En cuanto la interdisciplinariedad exige respetar la interacción entre los objetos de estudios de las diferentes disciplinas y lograr la interacción de sus aportes respectivos en un todo coherente y lógico, este postulado conlleva a la aceptación de que las realidades sistémicas se componen de elementos o constituyentes heterogéneos y son lo que son por su posición o por la función que desempeñan en la estructura o sistema total. Por tanto, no son aditivas ni conmutativas; requieren conceptos e instrumentos adecuados a su propia naturaleza (Capra, 1992). 
¿Transdisciplinar por qué? Porque la propia realidad reclama la transcendencia de la disciplina. No se trata del conocimiento fragmentado; por el contrario, de lo que se trata es de explicar y comprender la realidad desde distintas ópticas, integrándolas, rebasando la disciplina en sí, para dar respuesta a la problemática o la situación observada, la cual no puede ser conocida desde un enfoque único (Carvajal, 2010).

Si todo está estrictamente cohesionado, todo es sistémico. Entonces toda esa práctica implica una recreación en la gestión del conocimiento de acuerdo a la relación, de acuerdo a lo cotidiano, de acuerdo a cómo vaya surgiendo la historia. Ello implica una nueva manera para conocer, valorar e interpretar el mundo, y conlleva a una transformación de nuestro modo de descifrar lo "real". Sin lugar a dudas un cambio de paradigma. Es el uso del pensamiento divergente, la discrepancia razonada, la oposición lógica, la crítica fundada (Carvajal, 2011).

A fin de cuentas, somos constituyentes de un universo sistémico, que puede comprenderse con un enfoque estructural, dialéctico, inter- y transdisciplinar. Ahora bien, ¿cómo lograr insertar está concepción inter- y transdisciplinar en la gestión del conocimiento universitario? En la siguiente sección se marca la ruta, y se presentan los constructos de co-inspiración, mente sustentable, autopoiesis, y el principio de sustentabilidad para proponer el cambio hacia una gestión del conocimiento sustentable universitario (ver diagrama $\mathrm{N}^{\circ} 1$ ).

Diagrama 1: "Gestión del conocimiento sustentable como abstracción teórica"

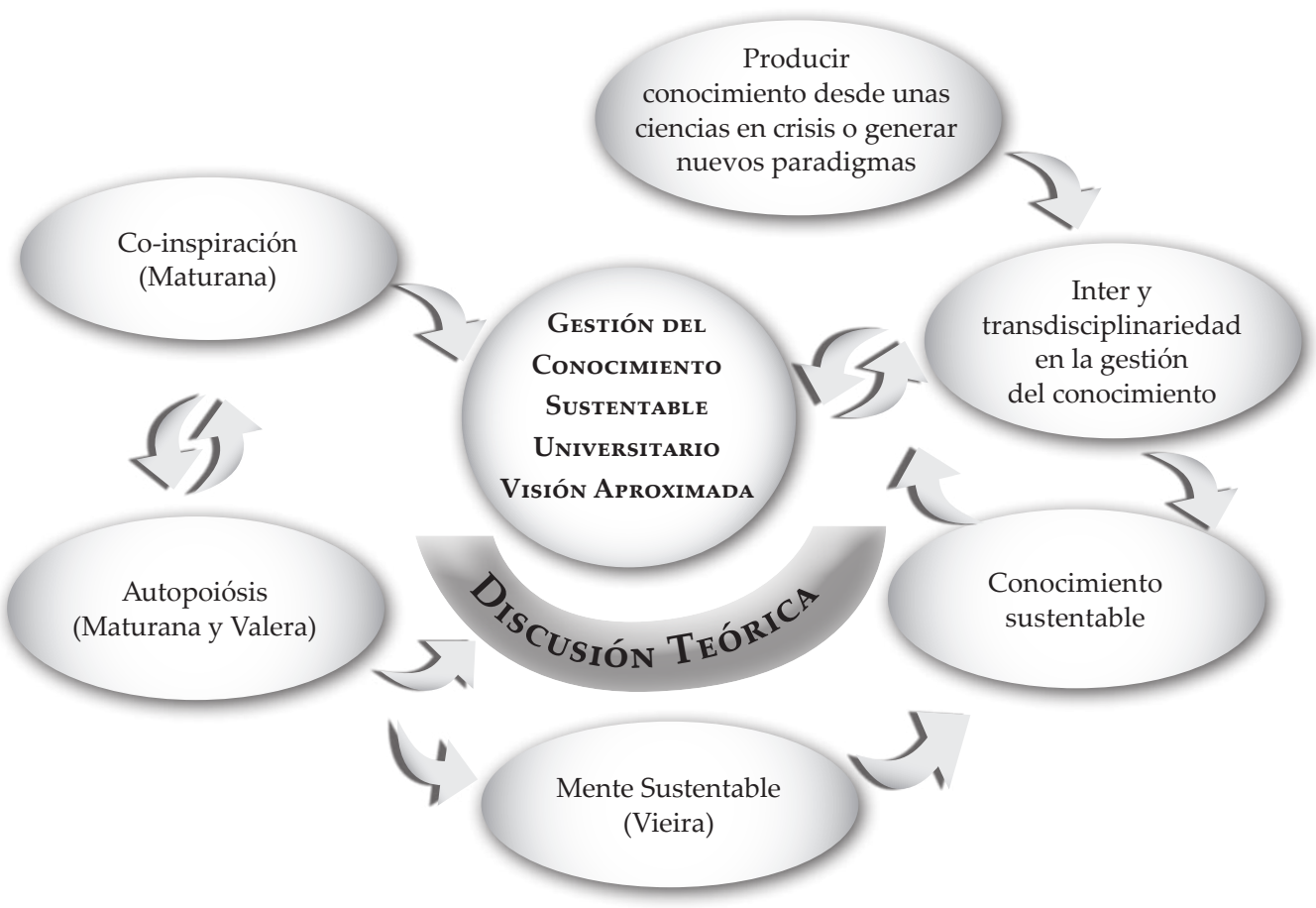

Fuente: Carvajal, 2014 


\section{Co-InSPIRACión (MATURANA)}

El constructo de co-inspiración lo propone el biólogo y epistemólogo chileno Humberto Maturana como un modo de observación de las relaciones humanas, en el que la impresión del amor moviliza los cambios en el entrelazamiento del lenguaje y la emoción, a lo que denomina: lenguajear. Sintetiza en una sola palabra una propuesta teórica, en la que presenta su reflexión acerca de los sistemas sociales humanos ${ }^{3}$.

La co-inspiración se da en una cultura matrística de respeto al otro como legítimo otro; la convivencia está centrada en redes de conversación fundadas en la estética de la armonía del mundo natural, en las conversaciones de comprensión, inclusión, acuerdo, respeto y compañerismo.

Para Maturana, los seres humanos, quienes existen en el lenguaje, pueden modificar cualquier situación desde la reflexión: al verse a sí mismos desde afuera, como especímenes que pueden darse cuenta de su contexto y seleccionar entre varias alternativas, escogiendo en función de las posibles consecuencias de lo que hacen.

Así, en un sistema social reflexivo, las interacciones conversacionales generadas en la co-inspiración bajo la hipótesis de la cultura matrística se contraponen a un modo de vida de lucha y de competencia, en el que la negación del otro nos reduce a ser agresivos y competitivos o fracasados y sumisos. Se contrapone a una cultura patriarcal, en

3 "Un sistema social es un sistema cerrado que incluye como sus miembros a todos aquellos organismos que operan bajo la emoción de la aceptación mutua en la realización de la red de coordinaciones de acciones que lo realizan" (Maturana, 1997a, pp. 93). la que se ejercen el control y el miedo como mecanismos regulatorios de una convivencia de premiación y castigo.

Como seres reflexivos en las UPL tenemos la opción de seleccionar una de estas culturas en la gestión del conocimiento. Es decir que podemos trabajar en redes sociales, desde una cultura centrada en la co-inspiración, reguladas en interacciones recurrentes de aceptación, compañerismo, autonomía y respeto, y en la armonía del convivir en las diferencias, o por el contrario, ceñirnos a una cultura de obediencia, control y temor.

\section{EL PRINCIPIO DE MENTE SUSTENTABLE (VIEIRA)}

Con este constructo, creado por el brasileño Evandro Vieira, se postula la reivindicación de la "razón sensible" (Vieira, 2009, 2011). El ser humano haría consciencia de que no sólo forma parte de la naturaleza, él es naturaleza. Desde este punto de vista, el desarrollo económico se sostiene en el trípode tierra-humanidad-desarrollo como sistema interconectado, fundando en la premisa de que el uso de la naturaleza tiene consecuencias que pueden ser devastadoras para la humanidad, porque impacta la totalidad.

Para Vieira es necesaria la revisión de esa relación, en cuanto conlleva a la observación de la pulsación de vida que ocurre en el intercambio hombre-naturaleza. En esta revisión cobra importancia la comunicación, cuando nos conectamos con lo que estamos haciendo, con lo que hemos estado creando, es "libertad y vinculación socioambiental" (Vieira, 2009, pp. 77). 
Vieira alude a la manifestación de la consciencia como experiencia de inspiración, de respeto por el orden natural, en la que se pasa de la oposición dualista a la complementariedad no dualista, en la cual se requiere un estado mental (pensamientos, afectos, sentimientos, emociones, percepciones, entre otros) en relación con un poder, un estado mental en donde el ser es uno con el ambiente, uno con la tierra que lo acoge.

Y cuanto más se realice una gestión mayor social y ecológicamente, más pensada en la conexión hombre-naturaleza, sería vista en conjunción, en forma rizomática, en algún sentido se diría que anónima, en el entendido de no egocéntrica. A ser uno con el ambiente, con la naturaleza, a ser uno con la tierra que nos acoge, esto implica un estado mental en relación con un poder; con ese que deviene de nosotros, seres humanos, en relación con el ambiente y aceptando las consecuencias de esa relación.

\section{AutopoIESIS Y EL SUPUESTO DE SUSTENTABILIDAD}

En este aparte se presenta el concepto de autopoiesis, para cerrar la disertación con la propuesta propia de sustentabilidad, en la gestión de conocimiento universitario. Se considera que el constructo autopoiesis - visto como principio explicatorio de patrones de vida - puede ser utilizado en todo sistema vivo. En este caso estamos definiendo a las universidades como sistema formado por constituyentes vivos, que están generando un conocimiento que puede ser sustentable o no para las generaciones futuras.
De tal manera, que si tomamos la tesis básica dada por Maturana y Valera (1999) de autopoiesis, tenemos como definición que es un patrón general de organización, común a todos los sistemas vivos, cualquiera que sea la naturaleza de sus componentes. La estructura está constituida por las propias relaciones entre los componentes físicos.

La comprensión de la autopoiesis está centrada en el patrón de organización de los sistemas vivos. La función de cada componente es participar en la producción o transformación de otros, como se produce a sí misma continuamente, ser y hacer son inseparables, de tal manera que los componentes de la red se generan de sí, es decir desde adentro. Por esto, todo sistema vivo es un patrón de red.

Las propiedades de una red autopoiética se definen de la siguiente manera: el sistema debe ser autolimitado, autogenerado y autoperpetuante. En otros términos: a) autolimitado en su extensión como constituyente de la red; b) autogenerado: todos los componentes del sistema hasta sus límites son producto de la red, y c) autoperpetuante: los procesos de producción perduran en el tiempo, de modo que todos los componentes son continuamente modificados por los procesos de transformación del sistema (Prigogine y Stengers, 1986).

Si la autopoiesis es definida como un patrón en red, en el que la función de cada componente es participar en la producción o transformación de otros componentes, entonces podemos aplicar este principio al desarrollo de una gestión de conocimiento sustentable en la universidad latinoamericana. Porque al formarse puntos de bifurcación, por las mismas presiones del entorno, 
nuevas formas de desorden pueden surgir espontáneamente, originando el desarrollo y la evolución. Esto generaría un cambio en la estructura del sistema: en este caso, de la universidad como sistema.

Una organización como la universidad, que es vista como sistema vivo, generará de igual manera bifurcaciones en las respuestas ante el entorno, las cuales afectarán su comportamiento futuro. Este comportamiento puede a su vez crear una respuesta, en la que se consideren la articulación de diversas teorías, disciplinas y ramas científicas que conlleven a la sustentabilidad del conocimiento (ver diagrama 2).
La gestión del conocimiento universitario ha sido una de las mayores preocupaciones de los académicos (Rivera, 2000; Espinoza, 1999; Sander, 1996) en estas últimas décadas, y el mismo cobra diversas especificidades en los diferentes claustros universitarios latinoamericanos. Factores de orden socio-económico, político y ecológico, tales como pobreza, hambre, desigual distribución del ingreso global, indisponibilidad de recursos médicos y biotecnológicos y daño ecológico, sólo por mencionar algunos, representan una realidad que impulsa a los académicos hacia la búsqueda de respuestas decisivas.

Diagrama 2: "Síntesis de interpretación teórica"

\section{Supuestos Teóricos}

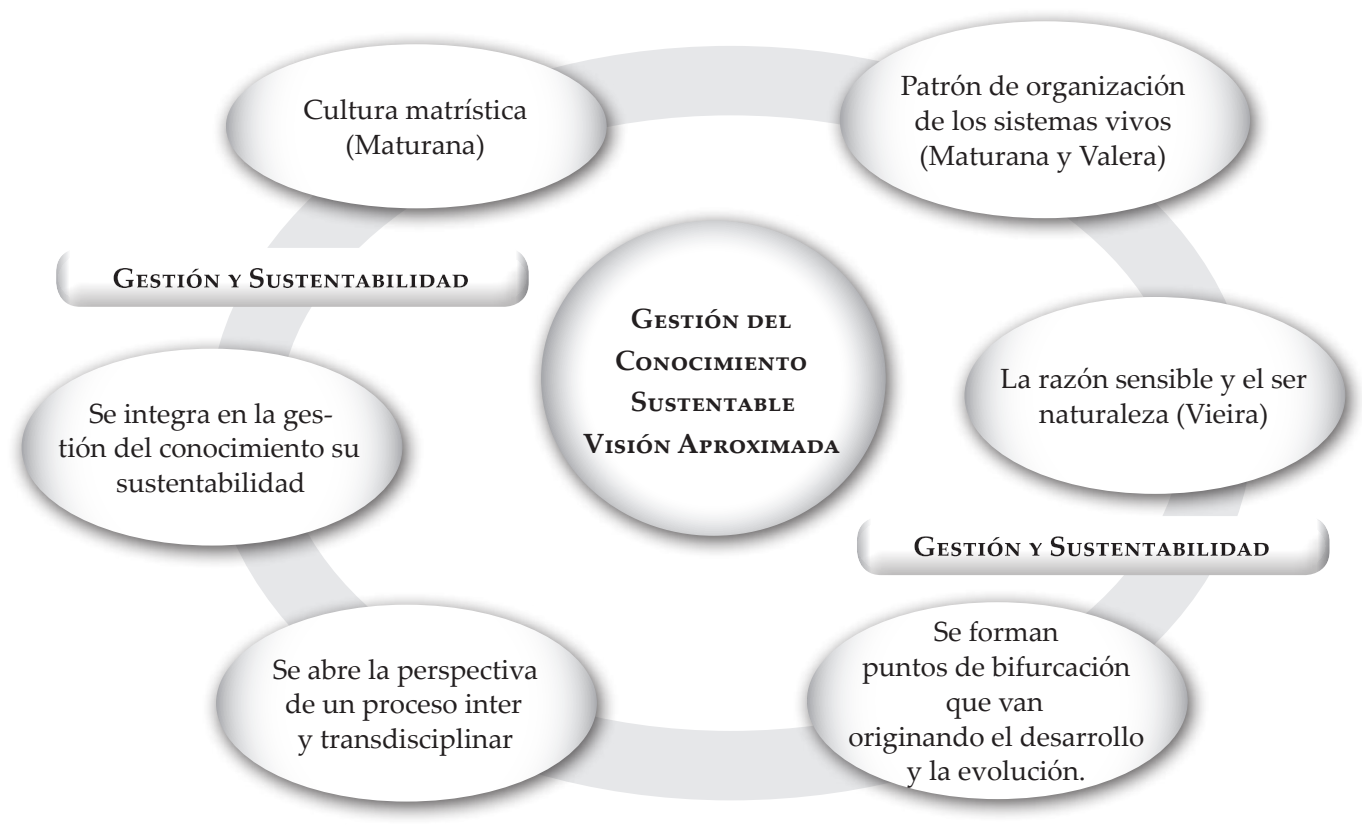

Fuente: Carvajal, 2014. 
Por consiguiente, en este artículo se incorpora el concepto de sustentabilidad al de gestión del conocimiento, y se plantea la pertinencia de una visión reflexiva acerca del compromiso asumido por la academia latinoamericana, con el modo de realizar la gestión del conocimiento. En este sentido, la sustentabilidad es concebida como proceso de cambio en el cual la explotación de los recursos, la dirección de las inversiones, la orientación del desarrollo tecnológico y el cambio institucional son fundamentales para incrementar el potencial, presente y futuro para la satisfacción de las necesidades y aspiraciones humanas.

La premisa de sustentabilidad implicaría la adopción de ajustes dinámicos con relación a factores institucionales (el estado, la comunidad, entre otros.), factores económicos y factores tecnocientíficos. No obstante, implicaría sobre todo una diferenciación entre necesidades y aspiraciones, lo cual obliga a una mayor coherencia entre el deber ser, el ser y el que hacer académico.

En efecto, se pone en relieve que la atención a las demandas del entorno pasa por aceptar que están fuertemente entrelazadas con valoraciones éticas, políticas e ideológicas, que orientan la formulación de programas y proyectos de investigación científica y tecnológica en la consecución de nuevos estilos de desarrollo, en el descubrimiento de fuentes alternativas de energía, en la innovación de tecnologías apropiadas, en el aprovechamiento racional de los recursos, fundados en las condiciones de productividad, estabilidad y de regeneración de los ecosistemas, y en la implementación de nuevas formas de organización productiva para la gestión social de los recursos de las comunidades (Leff, 1994).
Se estaría considerando esta visión de sustentabilidad, no sólo para entender la complejidad del conocimiento que se genera en los espacios universitarios, sino para comprender y analizar los elementos de la cultura donde nacen, porque para que en la vinculación universidad y entorno se logren niveles de mayor sustentabilidad, se requiere que el proceso de intercambio de conocimiento sea el resultante de una transformación de los paradigmas teóricos de las disciplinas involucradas, es decir, la construcción de una nueva forma de conocer, en la que se transcienda al objeto mismo de las disciplinas y se trabaje con una transdisciplinariedad del saber científico.

\section{Resultados}

Para el logro del objetivo de investigación que precedió este artículo, se realizó una pesquisa que incluyó entrevistas a un grupo de académicos de siete universidades latinoamericanas, situadas en Venezuela, Argentina y Brasil. En dichas entrevistas, se pulsó la opinión acerca de la gestión del conocimiento en sus instituciones de origen (universidad en la cual trabajaban), siempre contextualizando las respuestas en la localidad y país donde residen.

Como resultante de esas entrevistas, se agruparon las respuestas de los informantes en función de los puntos clave en los cuales se iba concentrando el mayor cúmulo de opinión, se categorizó ese resultado en cuatro aspectos generales, a saber: económico, organizacional, formación e investigación, y social, a partir de ello, se perfilaron categorías más específicas (ver cuadro 1), que dan cuenta de las opiniones recolectadas en el compendio presentado en los cuadros 2, 3, 
4 y 5. Posteriormente, en correspondencia con la información recabada y la reflexión de la teoría previamente expuesta, se despliegan los cuadros $6,7,8$ y 9 , en los cuales se desarrollan los lineamientos generales por categoría general de análisis, en función de las tendencias hacia donde converge la muestra total.

Cuadro 1: categorías de análisis

\begin{tabular}{|c|c|c|c|}
\hline ECONÓMICo & ORGANIZACIONAL & FORMACIÓN E INVESTIGACIÓN & Social \\
\hline $\begin{array}{l}\text { Presupuesto y gestión } \\
\text { del conocimiento. }\end{array}$ & $\begin{array}{l}\text { Cultura organizacional } \\
\text { para la gestión del } \\
\text { conocimiento. }\end{array}$ & $\begin{array}{l}\text { Relación producción } \\
\text { del conocimiento- } \\
\text { ambiente. }\end{array}$ & $\begin{array}{l}\text { Apropiación } \\
\text { social } \\
\text { del conocimiento }\end{array}$ \\
\hline $\begin{array}{l}\text { Demanda del entorno } \\
\text { socio-productivo } \\
\text { y la respuesta de } \\
\text { las universidades. }\end{array}$ & & $\begin{array}{l}\text { Inter y } \\
\text { transdisciplinariedad } \\
\text { en la gestión } \\
\text { del conocimiento }\end{array}$ & \\
\hline
\end{tabular}

Fuente: entrevista semi-estructurada a informantes clave (Carvajal, 2013).

Cuadro 2: Opinión por categoría de análisis (económicas)

\begin{tabular}{|c|c|c|c|}
\hline País / Categoría & Venezuela & Brasil & Argentina \\
\hline $\begin{array}{l}\text { Presupuesto } \\
\text { y gestión del } \\
\text { conocimiento }\end{array}$ & $\begin{array}{l}\text { El presupuesto universitario } \\
\text { no ha sufrido ningún ajuste } \\
\text { en los últimos años. Esto } \\
\text { trae como consecuencia } \\
\text { que las labores propias de } \\
\text { la universidad y que están } \\
\text { en estrecha relación con } \\
\text { la sustentabilidad del } \\
\text { conocimiento se vean } \\
\text { afectadas, principalmente } \\
\text { las relacionadas con } \\
\text { investigación y extensión. } \\
\text { El presupuesto de las } \\
\text { universidades se consume } \\
\text { en sueldos y compromisos } \\
\text { a proveedores. Con una } \\
\text { institución marcada financie- } \\
\text { ramente por estas partidas, } \\
\text { muy poco se puede esperar. } \\
\text { Los informantes de las dos } \\
\text { universidades experimenta- } \\
\text { les coinciden en afirmar que } \\
\text { en los últimos } 12 \text { años } \\
\text { (a partir del año 2000) se } \\
\text { ha entendido la gestión } \\
\text { universitaria como un pro- } \\
\text { ceso de imposición, el cual } \\
\text { ha afectado la generación e } \\
\text { innovación del conocimiento. }\end{array}$ & $\begin{array}{l}\text { Existen realidades } \\
\text { divergentes a lo interno } \\
\text { de cada universidad, } \\
\text { aunque se advierte que } \\
\text { la mejoría relativa de } \\
\text { la economía brasileña } \\
\text { en la última década se } \\
\text { ha traducido en mejoras } \\
\text { de los presupuestos } \\
\text { universitarios y, conse- } \\
\text { cuentemente, en acciones } \\
\text { concretas que pueden } \\
\text { haber propiciado } \\
\text { la generación de un } \\
\text { conocimiento sustentable. } \\
\text { No obstante la tendencia } \\
\text { debería ser hacia } \\
\text { la autogestión-los infor- } \\
\text { mantes concuerdan en } \\
\text { que las universidades } \\
\text { públicas no pueden } \\
\text { depender tanto del } \\
\text { gobierno, porque entonces } \\
\text { hay una especie de } \\
\text { acorralamiento hacia } \\
\text { la institución y la univer- } \\
\text { sidad, a fin de cuentas } \\
\text { es la que produce y } \\
\text { genera el conocimiento. }\end{array}$ & $\begin{array}{l}\text { Desde el año } 2003 \text { la } \\
\text { universidad retomó } \\
\text { un papel protagónico, } \\
\text { a partir de una } \\
\text { progresiva y soste- } \\
\text { nida asignación de } \\
\text { recursos. La univer- } \\
\text { sidad pública ha sido } \\
\text { beneficiada por po- } \\
\text { líticas decisivas para } \\
\text { impulsar la investiga- } \\
\text { ción y el desarrollo. } \\
\text { En este contexto, se ha } \\
\text { avanzado en diversos } \\
\text { programas que favo- } \\
\text { recen la investigación } \\
\text { básica y aplicada y, } \\
\text { especialmente, el } \\
\text { trabajo interdisciplina- } \\
\text { rio y la difusión } \\
\text { del conocimiento } \\
\text { (por medio de publica- } \\
\text { ciones científicas, } \\
\text { de divulgación, etc.). } \\
\text { No obstante, todavía } \\
\text { la asignación de } \\
\text { recursos sigue siendo } \\
\text { insuficiente: esto } \\
\text { debe profundizarse. }\end{array}$ \\
\hline
\end{tabular}

Fuente: entrevista semi-estructurada a informantes clave (Carvajal, 2013). 


\begin{tabular}{|c|c|c|c|}
\hline $\begin{array}{c}\text { País } \\
\text { Categoría }\end{array}$ & Venezuela & Brasil & Argentina \\
\hline $\begin{array}{l}\text { Demanda } \\
\text { del entorno } \\
\text { socio-Çpro- } \\
\text { ductivo } \\
\text { y la respuesta } \\
\text { de las } \\
\text { universidades }\end{array}$ & $\begin{array}{l}\text { Los profesionales } \\
\text { egresados de nuestras } \\
\text { universidades son de } \\
\text { excelente calidad. Los } \\
\text { egresados de las universi- } \\
\text { dades autónomas, son } \\
\text { en términos generales } \\
\text { competitivos fuera y den- } \\
\text { tro del país. La gestión de } \\
\text { conocimiento sustentable, } \\
\text { la reproducción del } \\
\text { conocimiento a través } \\
\text { de la docencia está } \\
\text { asegurada. No obstante, } \\
\text { al ocupar la docencia el } \\
\text { mayor tiempo y esfuerzo } \\
\text { de los profesores univer- } \\
\text { sitarios, las demandas del } \\
\text { sector socioproductivo } \\
\text { se cubren en la inmediatez } \\
\text { del recurso humano, } \\
\text { no así en la investigación } \\
\text { aplicada, mucho menos } \\
\text { en la básica. }\end{array}$ & $\begin{array}{l}\text { La universidad pública } \\
\text { en los actuales momentos } \\
\text { presenta una capacidad } \\
\text { de competencia minimi- } \\
\text { zada en relación con las } \\
\text { universidades privadas. } \\
\text { Y uno de los graves } \\
\text { problemas que enfrentan } \\
\text { es la falta de presupues- } \\
\text { tos que garanticen mayor } \\
\text { y actualizada investiga- } \\
\text { ción. Las demandas del } \\
\text { entorno son mayores: } \\
\text { la pobreza, el hambre, } \\
\text { las desigualdades del } \\
\text { desarrollo económico, } \\
\text { entre otras; pero cómo } \\
\text { responder si no se tiene } \\
\text { la posibilidad de ingresos } \\
\text { para hacer una educación } \\
\text { más actualizada con } \\
\text { la producción de las } \\
\text { ciencias en el nivel } \\
\text { mundial. }\end{array}$ & $\begin{array}{l}\text { La universidad pública en } \\
\text { Argentina, si se consideran } \\
\text { la últimas tres décadas, ha } \\
\text { estado orientada en su relación } \\
\text { con el entorno socioproductivo } \\
\text { de manera ciclotímica. Las } \\
\text { Universidad, a pesar de tener } \\
\text { bajo su responsabilidad la } \\
\text { investigación, y con ello } \\
\text { la producción de tecnología } \\
\text { para el entorno no termina de } \\
\text { gestionar de manera proactiva } \\
\text { hacia ese entorno. Entonces } \\
\text { lo que sucede es una tendencia } \\
\text { hacia el desarrollo de investi- } \\
\text { gación más en corresponden- } \\
\text { cia de grupos de investiga- } \\
\text { dores, a través de un gran } \\
\text { esfuerzo personal, pero que } \\
\text { no termina de acercarnos } \\
\text { a una producción científica } \\
\text { con miras a ubicarnos como } \\
\text { universidades de vanguardia } \\
\text { en América Latina. }\end{array}$ \\
\hline
\end{tabular}

Fuente: entrevista semi-estructurada a informantes clave (Carvajal 2013).

Cuadro 3: Opinión por categoría de análisis (organizacionales)

\begin{tabular}{|c|c|c|c|}
\hline $\begin{array}{c}\text { País } \\
\text { Categoría }\end{array}$ & Venezuela & Brasil & Argentina \\
\hline $\begin{array}{l}\text { Cultura } \\
\text { organizacional } \\
\text { para la } \\
\text { gestión del } \\
\text { conocimiento }\end{array}$ & $\begin{array}{l}\text { En estos recientes } \\
\text { catorce años las } \\
\text { universidades } \\
\text { experimentales -que } \\
\text { dependen en su estructura } \\
\text { organizativa del gobierno } \\
\text { central-han sido } \\
\text { intervenidas, mientras, } \\
\text { las universidades } \\
\text { autónomas se } \\
\text { encuentran bajo } \\
\text { amenazas de su } \\
\text { autonomía. Contexto } \\
\text { queha repercutido en } \\
\text { su cultura organizacional } \\
\text { y en la gestión del conoci- } \\
\text { miento en particular. } \\
\text { Como consecuencia: } \\
\text { la cultura de la urgencia } \\
\text { como antinomia }\end{array}$ & $\begin{array}{l}\text { Muy poco a poco, las } \\
\text { cosas están mudando. } \\
\text { Más estas cosas } \\
\text { son de aspectos } \\
\text { culturales, de } \\
\text { cultura de la propia } \\
\text { organización. } \\
\text { La disyuntiva principal } \\
\text { es: cómo reproducir } \\
\text { un conocimiento como } \\
\text { profesor universitario, } \\
\text { sino se procura, se } \\
\text { debate, se comprueba } \\
\text { y se refuta. Entonces } \\
\text { la universidad } \\
\text { está valorando y } \\
\text { estimulando la cultura } \\
\text { para la investigación } \\
\text { y con ello la gestión } \\
\text { de un conocimiento }\end{array}$ & $\begin{array}{l}\text { Esta sigue siendo } \\
\text { una tarea pendiente } \\
\text { en la mayoría de las } \\
\text { universidades argentinas. } \\
\text { Se observa, a lo interno } \\
\text { de algunas unidades } \\
\text { académicas e investigativas } \\
\text { una fuerte preocupación } \\
\text { por la forma como se } \\
\text { gestiona el conocimiento, } \\
\text { principalmente en } \\
\text { macro universidades } \\
\text { como la Universidad } \\
\text { de Buenos Aires (UBA) } \\
\text { y la Universidad } \\
\text { de Córdoba (UC). } \\
\text { Se coincide que } \\
\text { los cambios en la cultura } \\
\text { organizacional para la } \\
\text { gestión del conocimiento }\end{array}$ \\
\hline
\end{tabular}




\begin{tabular}{|c|c|c|c|}
\hline $\begin{array}{c}\text { País } \\
\text { Categoría }\end{array}$ & Venezuela & Brasil & Argentina \\
\hline $\begin{array}{l}\text { Cultura } \\
\text { organizacional } \\
\text { para la } \\
\text { gestión del } \\
\text { conocimiento }\end{array}$ & $\begin{array}{l}\text { a la cultura de } \\
\text { la sustentabilidad, } \\
\text { inves-tigadores } \\
\text { trabajando en forma } \\
\text { individualizada o } \\
\text { en grupos pequeños } \\
\text { con intereses comunes. } \\
\text { Se refleja en esta praxis } \\
\text { solo el trabajo de un } \\
\text { grupo o individualidad } \\
\text { que lo gestiona. }\end{array}$ & $\begin{array}{l}\text { más sustentable. } \\
\text { Eso es lo que se hace } \\
\text { a lo interno, pero } \\
\text { debe guardar más } \\
\text { correspondencia } \\
\text { con el Estado, con } \\
\text { la sociedad civil; } \\
\text { que estos sepan } \\
\text { lo que se está haciendo. } \\
\text { Lo universitarios } \\
\text { no pueden quedarse } \\
\text { en la retaguardia. }\end{array}$ & $\begin{array}{l}\text { ocurren, principalmente, } \\
\text { en unidades con mayor } \\
\text { grado de autonomía } \\
\text { como la de Estudios } \\
\text { Avanzados (UC), no } \\
\text { así en el nivel de pregrado } \\
\text { donde aún se depende } \\
\text { de una cultura mediada } \\
\text { por la inmediatez. }\end{array}$ \\
\hline
\end{tabular}

Fuente: entrevista semi-estructurada a informantes clave (Carvajal, 2013).

Cuadro 4: Opinión por categoría de análisis (formación e investigación)

\begin{tabular}{|c|c|c|c|}
\hline $\begin{array}{c}\text { País } \\
\text { Categoría }\end{array}$ & Venezuela & Brasil & Argentina \\
\hline $\begin{array}{l}\text { Relación } \\
\text { producción del } \\
\text { conocimiento- } \\
\text { ambiente }\end{array}$ & $\begin{array}{l}\text { La universidad pública } \\
\text { venezolana, a pesar } \\
\text { de ser una de las pocas } \\
\text { instituciones que aún } \\
\text { se mantienen realizando } \\
\text { investigación actualmente } \\
\text { en el país, ha ido } \\
\text { perdiendo en los últimos } \\
\text { años su capacidad de } \\
\text { gestionar conocimiento. } \\
\text { La universidad está } \\
\text { asfixiada entre políticas } \\
\text { públicas que le han } \\
\text { venido coartando ese } \\
\text { vínculo de generación } \\
\text { de conocimiento } \\
\text { en y para la gestión } \\
\text { ambiental. La posibilidad } \\
\text { de transferencia de } \\
\text { conocimiento en el área } \\
\text { ambiental está soportada } \\
\text { en individualidades } \\
\text { o pequeños grupos } \\
\text { de investigadores, } \\
\text { que con un gran } \\
\text { esfuerzo personal, } \\
\text { producen y divulgan } \\
\text { los resultados } \\
\text { de sus estudios. }\end{array}$ & $\begin{array}{l}\text { Lo que acontece en } \\
\text { nuestras políticas públicas } \\
\text { y de ellas devienen las } \\
\text { educativas, es que el } \\
\text { orden natural (se refieren } \\
\text { al ecológico) respetado } \\
\text { por los ancestros es } \\
\text { saltado cuando solo } \\
\text { se fundamenta en el } \\
\text { razonamiento, y ese } \\
\text { orden natural en } \\
\text { que el ambiente es } \\
\text { el elemento nodal, } \\
\text { es, más bien, puesto } \\
\text { en último lugar. } \\
\text { Así la producción } \\
\text { de conocimiento } \\
\text { en las universidades } \\
\text { está enraizada en } \\
\text { la razón Y nada funciona } \\
\text { de manera natural, } \\
\text { la naturaleza es } \\
\text { obligada a ser, a } \\
\text { comportarse de la } \\
\text { manera que esperamos. } \\
\text { La premisa que prevalece } \\
\text { es que los seres humanos } \\
\text { tienen el control absoluto } \\
\text { del entorno natural. } \\
\text { La base del trabajo } \\
\text { realizado no es ecológica. }\end{array}$ & $\begin{array}{l}\text { La ecuación } \\
\text { conocimiento= } \\
\text { preservación } \\
\text { del medio ambiente } \\
\text { no es una realidad } \\
\text { tangible en la } \\
\text { praxis investigativa } \\
\text { universitaria. } \\
\text { Esto no significa } \\
\text { que no se realicen } \\
\text { investigaciones en } \\
\text { procura del desarrollo } \\
\text { sustentable, de } \\
\text { hecho se adelantan } \\
\text { en las facultades } \\
\text { de agronomía, } \\
\text { veterinaria, y } \\
\text { educación importantes } \\
\text { trabajos en y para } \\
\text { el desarrollo } \\
\text { sostenible y la } \\
\text { agricultura orgánica; } \\
\text { no obstante } \\
\text { considerar que en } \\
\text { la macro-estructura } \\
\text { prevalece un } \\
\text { pensamiento } \\
\text { ecologista } \\
\text { es incierto. }\end{array}$ \\
\hline
\end{tabular}




\begin{tabular}{|c|c|c|c|}
\hline $\begin{array}{c}\text { País } \\
\text { Categoría }\end{array}$ & Venezuela & Brasil & Argentina \\
\hline $\begin{array}{l}\text { Inter- y } \\
\text { transdiscipli- } \\
\text { nariedad en la } \\
\text { gestión } \\
\text { del conocimiento }\end{array}$ & $\begin{array}{l}\text { La interdisciplinariedad } \\
\text { funciona en esos pequeños } \\
\text { grupos de excelencia } \\
\text { académica que se mantienen, } \\
\text { a pesar de las circunstancias, } \\
\text { a la vanguardia de las } \\
\text { exigencias de la ciencia. Pero } \\
\text { en líneas generales los espacios } \\
\text { de producción intelectual } \\
\text { se han reducido al nivel } \\
\text { de lo personal, no hay } \\
\text { investigación ni inter-, } \\
\text { ni transdisciplinar y la } \\
\text { investigación avanza de } \\
\text { acuerdo a los requerimientos } \\
\text { individuales del personal do- } \\
\text { cente, impactando, a lo sumo, } \\
\text { la práctica académica de cada } \\
\text { profesor en el seno de sus } \\
\text { cátedras y esto es una gran } \\
\text { debilidad tanto en el marco } \\
\text { de las respuesta que el } \\
\text { entorno socio-productivo } \\
\text { demanda, como en el } \\
\text { grado de competencia ante } \\
\text { el desarrollo de las ciencias } \\
\text { en general. }\end{array}$ & $\begin{array}{l}\text { Aun cuando prevalece } \\
\text { una praxis parcelaria } \\
\text { y disciplinar del } \\
\text { conocimiento, sí } \\
\text { se vienen haciendo } \\
\text { importantes avances } \\
\text { en el trabajo en red, } \\
\text { lo cual conlleva } \\
\text { una comunicación } \\
\text { interdisciplinar } \\
\text { para dar paso a una } \\
\text { investigación } \\
\text { proactiva, polifuncional, } \\
\text { y en muy pocos casos, } \\
\text { transdisciplinar. } \\
\text { Se intenta superar } \\
\text { los estancos } \\
\text { académicos para, } \\
\text { así, impactar en el } \\
\text { quehacer investigativo } \\
\text { de los estudiantes. } \\
\text { Es eso lo que se está } \\
\text { buscando. Más allá del } \\
\text { trabajo colaborativo } \\
\text { entre los profesores, } \\
\text { es el trabajo en red } \\
\text { interdisciplinar. }\end{array}$ & $\begin{array}{l}\text { Se dan casos } \\
\text { específicos } \\
\text { de políticas } \\
\text { institucionales } \\
\text { en gestión de la } \\
\text { interdisciplinariedad } \\
\text { en investigación } \\
\text { y posgrado, } \\
\text { se favorece el } \\
\text { desarrollo de } \\
\text { programas y proyectos } \\
\text { en los que participan } \\
\text { distintas facultades. } \\
\text { A pesar de lo señalado, } \\
\text { todavía debe } \\
\text { considerarse } \\
\text { que en la universidad } \\
\text { el trabajo inter y } \\
\text { transdisciplinario } \\
\text { encuentra mucha } \\
\text { resistencia y puede } \\
\text { exhibir sólo algunos } \\
\text { ejemplos aislados. }\end{array}$ \\
\hline
\end{tabular}

Fuente: entrevista semi-estructurada a informantes clave, (Carvajal 2013).

Cuadro 5: Opinión por categoría de análisis (social)

\begin{tabular}{|c|c|c|c|}
\hline $\begin{array}{c}\text { País } \\
\text { Categoría }\end{array}$ & Venezuela & Brasil & Argentina \\
\hline $\begin{array}{l}\text { Apropiación social } \\
\text { del conocimiento }\end{array}$ & $\begin{array}{l}\text { En nuestro país, hablar } \\
\text { de desarrollo basado } \\
\text { en la cualificación } \\
\text { de la educación } \\
\text { universitaria resulta } \\
\text { utópico en este } \\
\text { contexto sociopolítico. } \\
\text { La generación de } \\
\text { conocimiento como } \\
\text { única fuente de } \\
\text { ventaja competitiva } \\
\text { sostenible, para } \\
\text { la población, y su } \\
\text { impacto en conjunto, } \\
\text { en los problemas } \\
\text { sociales, económicos, } \\
\text { ambientales y } \\
\text { culturales, }\end{array}$ & $\begin{array}{l}\text { Se observa una tendencia } \\
\text { de las universidades } \\
\text { a mantener el } \\
\text { conocimiento dentro } \\
\text { de sus muros. Y a pesar } \\
\text { de que se hacen intentos } \\
\text { por establecer esa } \\
\text { cooperación población- } \\
\text { universidad está faltando } \\
\text { afianzar la relación } \\
\text { necesaria del profesor } \\
\text { y los estudiantes con el } \\
\text { ciudadano de a pie. Por } \\
\text { ejemplo: la gente quiere } \\
\text { saber por qué es abusada } \\
\text { en su trabajo, por qué la } \\
\text { violencia en las calles, y } \\
\text { no tiene respuesta a eso. }\end{array}$ & $\begin{array}{l}\text { La universidad } \\
\text { ha dado algunos pasos } \\
\text { importantes en lo que se } \\
\text { conoce como apropiación } \\
\text { social del conocimiento, } \\
\text { que sucintamente } \\
\text { promueve una } \\
\text { comunicación más } \\
\text { horizontal entre la } \\
\text { universidad que genera } \\
\text { el conocimiento } \\
\text { y la sociedad que le } \\
\text { asigna tal función } \\
\text { y le demanda respuestas } \\
\text { a los problemas que } \\
\text { acucian a la propia } \\
\text { sociedad. }\end{array}$ \\
\hline
\end{tabular}




\begin{tabular}{|c|c|c|}
\hline $\begin{array}{c}\text { País } \\
\text { Categoría }\end{array}$ & Venezuela & Argentina \\
\hline & $\begin{array}{l}\text { se hace individual- } \\
\text { mente, o en micro- } \\
\text { grupos de excelencia } \\
\text { académica, que conti- } \\
\text { núan investigando y } \\
\text { transfiriendo conoci- } \\
\text { miento a pesar de la } \\
\text { crisis presupuestaria } \\
\text { que presenta hoy día } \\
\text { la universidad pública } \\
\text { venezolana. }\end{array}$ & $\begin{array}{l}\text { Los investigadores tien- } \\
\text { den entonces a trabajar } \\
\text { directo para el gobierno, } \\
\text { y entonces eso genera } \\
\text { una gran distancia con la } \\
\text { población en sí; porque } \\
\text { quien financia la inves- } \\
\text { tigación es el gobierno, } \\
\text { y por lo menos en la } \\
\text { parte social, los brasile- } \\
\text { ños no son escuchados } \\
\text { como ellos quieren ser } \\
\text { escuchados. }\end{array}$ \\
\hline
\end{tabular}

Fuente: entrevista semi-estructurada a informantes clave (Carvajal, 2013).

Cuadro 6: Lineamientos generales categoría de análisis: (económica)

\section{LINEAMIENTOS GENERALES}

Diversificar fuentes de financiamiento: la universidad latinoamericana podrá dar respuestas al entorno cambiante y contribuir a la gestión de conocimiento sustentable en la medida en que sus políticas estén orientadas a tal fin, para ello tendrían que desafiar su propia dependencia presupuestaria del gobierno central: la autogestión en una mayor contribución científico-técnica con el entorno productivo, suscribiendo programas de vinculación institucional que favorezcan la realización de actividades conjuntas, y el intercambio de estudiantes, docentes e investigadores.

Integrar redes: al estar las universidades integradas en redes por región se racionalizan los recursos económicos provenientes del estado y se fortalece la función académica, científica y social de la región y del país.

Ejercer autonomía responsable: una evaluación institucional que incluya programas académicos donde se creen condiciones para el ejercicio de la autonomía responsable y promueva condiciones para el fortalecimiento y desarrollo de la comunidad académica y de su proyección hacia la sociedad dentro de una concepción social abierta y flexible, y menos intervencionista por parte del estado

Fuente: (Carvajal 2014). 
Cuadro 7: Lineamientos generales categoría de análisis: organizacional

\section{LINEAMIENTOS GENERALES}

Generar una cultura organizacional fundada en el trabajo en equipos con alto grado de autonomía y libertad creativa y en las redes de comunicación.

Hacer coexistir como igualmente importantes y sin subordinación el uno del otro, dos focos de referencia: la organización universitaria como un todo y cada una de las personas que la constituyen. Este cambio estaría sustentado en una cultura organizacional co-inspirada para lo cual será necesario avanzar hacia una cultura organizacional matrística, de respeto al otro como legitimo otro; en una trama relacional intrauniversitaria en la que el compromiso del colectivo sea el que viabilice la transformación de esa red social. Se trabaja en una estructura matricial, descentralizada y departamentalizada, desde la cual se estaría accionando a través de principios de complementariedad, cooperación, competencia, en y para la organización. Todo este conjunto generaría una redefinición en la praxis de gestión del conocimiento universitario.

Transformar los patrones: en la autopoiesis la función de cada componente es participar en la producción o transformación de otros; de tal modo que al auto producirse, ser y hacer son inseparables en un patrón de red (Prigogine y Stengers, 1986). En la gestión del conocimiento sustentable universitario los diferentes componentes de la red, están representados por los distintos actores sociales que tanto en concordancia, como en divergencia generaría redes de cooperación que pudiera potenciar la concurrencia a lo interno de la organización, y con los sectores productivos y el estado.

Fuente: (Carvajal 2014).

Cuadro 8: Lineamientos generales categoría de análisis: formación e investigación

\begin{tabular}{l}
\hline LiNEAMIENTOS GENERALES \\
\hline Responder al entorno de manera inter- y transdisciplinar: es importante que en las universidades se creen \\
espacios de gestión que garanticen el desarrollo de competencias en el futuro profesional hacia la inter y \\
transdisciplinariedad, que le permitan ante los cambios de la sociedad, re-inventarse y ser competitivo en \\
lo laboral. Se está proponiendo una gestión que incentive no sólo el carácter vinculante entre las diferentes \\
disciplinas, sino que garantice la visión compleja y global de los problemas a los que se enfrentan los seres \\
sociales y el trabajo en equipo como una forma de hacer y ser laboralmente. \\
Trabajar en red: es importante que la universidad latinoamericana haga uso del potencial que tienen \\
las redes, las cuales se fortalecen con el uso de las tecnologías de información y comunicación. Estas \\
herramientas hacen que desaparezcan las barreras geográficas y pueda hacerse un intercambio masivo \\
de experiencias que contribuyan con la gestión del conocimiento sustentable. \\
Integrar el constructo de mente sustentable (Vieira, 2011) en la gestión del conocimiento universitario: debe \\
darse una profunda discusión en la que se inserte la dimensión ecológica que propicie su transversalidad, \\
tanto en la investigación como la docencia. Desde esta práctica se concretaría un estilo de gestión que \\
contemple la mejor calidad de vida de las comunidades, la posesión de bases tecnológicas respetuosa \\
de los principios ecológicos, la vinculación estrecha, constante y creativa con la tradición cultural de su \\
propio contexto.
\end{tabular}

Fuente: (Carvajal 2014). 
Cuadro 9: Lineamientos generales, categoría de análisis: social

\section{LINEAMIENTOS GENERALES}

Generar capacidades desde la propia organización universitaria para adaptarse con agilidad y eficiencia a los cambios y a las demandas de la sociedad formando para lo desconocido. Con una propuesta de educación permanente se crean oportunidades de actualización y adaptación a las realidades cambiantes y poco previsibles.

Cooperar: la cooperación educativa asumida como modalidad de gestión, en la que el empleador y la universidad comparten la responsabilidad social y económica en la formación del estudiante, es decir, se transfiere conocimiento de la universidad hacia el mundo del trabajo y se transfieren problemas y aprendizajes prácticos desde la empresa hacia la universidad.

Accionar la relación simbiótica universidad-sociedad: se trata de rescatar la vinculación a partir de una gestión de conocimiento que promueva la consecución de una educación de calidad y con transcendencia social que estaría asociada a la apropiación social del conocimiento.

Gestionar en mancomún: como trabajo cooperativo, que vaya más allá de un acto declarativo y demagógico, la vinculación de las universidades con su entorno social demandan de éstas respuestas al ciudadano de a pie. La transferencia de conocimiento pasa por la generación de conocimiento por y para las comunidades, subrayando en especial que la gestión debe contribuir al desarrollo sustentable y la mejor calidad de vida de los habitantes que forman parte del entorno socioproductivo.

Fuente: (Carvajal 2014).

\section{Conclusiones}

A continuación se presentan algunas conclusiones. El propósito es hacer una invitación abierta a la reflexión en torno a todos los aspectos interpretados en esta disertación:

- Una de las mayores preocupaciones de los académicos en estas últimas décadas ha sido la producción y gestión del conocimiento sustentable universitario, el cual cobra diversas especificidades en los diferentes claustros universitarios latinoamericanos. Factores como la pobreza, el hambre, las desigualdades del desarrollo económico, la distribución social de la producción, los costos ecológicos del crecimiento económico, las respuestas de la biotecnología y los avances en medicina son algunos de los elementos que movilizan constantemente a las universidades y sus respuestas al entorno demandante.

- Se plantea la urgencia de una búsqueda constante de la renovación en la gestión del conocimiento, la cual estaría precedida por un cambio fundado en la intery la transdisciplinariedad, y sustentada en una red conversacional de comprensión, inclusión, acuerdo: co-inspiración.

- El constructo de sustentabilidad lleva implícita la adopción de ajustes dinámicos, con relación a factores institucionales (el estado, la comunidad, entre otros), factores económicos y factores tecno-científicos, implica sobre todo una diferenciación entre necesidades y aspiraciones, 
referidas las necesidades al contexto de la pobreza mundial, y las aspiraciones a los patrones de vida básicos. El proceso de cambio que subyace en esta concepción incluye los ecosistemas, la demografía y las estructuras políticas.

- Visto así, el concepto de sustentabilidad aplicado a la necesidad de la gestión del conocimiento sustentable obliga al planteamiento de originales postulados y novedosas formas de acercamiento y compresión de una realidad, que no puede ni debe ser vista de igual modo, en todos y cada uno de los contextos históricossociales en los cuales se manifiesta.

- La gestión de un conocimiento sustentable fomenta la compresión y diagnóstico de múltiples procesos, que hacen imperativa la producción y aplicación del conocer transcientífico, el cual emerge como punto de inicio, para interpretar la creciente complejidad de objetos de estudios que tienen las ciencias en la actualidad. Ante tal diversidad, lo transdisciplinario se constituye en una vía que se sale de los encuadres paradigmáticos tradicionales, sustituyéndolo por una visión teórica-metodológica, que en un proceso interpretativo y que va en alternancia de la síntesis al análisis; del todo a las partes y de las partes al todo, donde las diferentes concepciones disciplinarias se entretejen de manera complementaria y dialéctica.

- Así, la dinámica del conocimiento universitario es analizada desde una concepción ecológica, esto es, considerando los efectos de ciertos fenómenos geofísicos (catástrofes naturales), de ciertos procesos atmosféricos (modificaciones del clima, inundaciones) y de ciertos procesos socio-históricos (modos de producción, prácticas productivas, fuerzas de producción), que afectan y transforman su comportamiento. Ello demanda la articulación de la geología, la geofísica, la historia, la antropología y la sociología, en conjunción con una ciencia que estudie los procesos ecosistémicos.

- Las relaciones personales como fuentes de vínculos, participación y compromiso con el cambio a lo interno de la organización universitaria; convivir en la diferencia, en la aceptación del otro como legítimo otro; desde este punto de vista ya no estaríamos anclados en una gestión del conocimiento que propicie la cultura individualista, más bien la propuesta es, trabajar en interconexiones que favorezcan el trabajo en red, en interdependencia.

\section{ReFERENCIAS}

Capra, F. (1992) El Tao de la Física (Juan José Alonso Rey, Tr.). Barcelona: Humanitas

Carvajal, B. (2011) Cultura investigativa universitaria. Una mirada desde la Universidad venezolana para favorecer su inter- $y$ transdisciplinariedad. En A. Robledo, M. Pisano, \& M. Paladini (Eds.), El estado de la investigación educativa. Perspectivas latinoamericanas (pp. 561-576). Argentina: Universidad Católica de Córdoba.

Carvajal, B. (2010). Inter- y transdisciplinariedad en la cultura investigativa universitaria. Cómo favorecer su práctica en la universidad venezolana. Revista Venezolana de Ciencias Sociales UNERMB, 14 (2), 164-178. 
Carvajal, B., y Carvajal, M. (2010). Universidad y entorno laboral: visión prospectiva de la educación superior desde la perspectiva de las competencias laborales. Revista Informe de Investigaciones Educativas, Vol. XXIV, pp. 27-50.

Carvajal, B. (2003). Aproximación prospectiva al estudio de escenarios para el cambio en la universidad pública venezolana. Heterotopia, 9(25), pp. 93-118.

Espinoza, R. (1999). Naturaleza y alcance de la relación universidad-sector productivo. Maracaibo: Universidad del Zulia.

Habermas, J. (2004). Tiempo de transiciones. Madrid: Trotta.

Leff, E. (1994). Racionalidad ambiental, democracia participativa y desarrollo sustentable. México: Siglo XXI.

Maturana, H. (1997a). La objetividad. Un argumento para obligar. Santiago de Chile: Dolmen.

Maturana, H. (1997b). Emociones y Lenguaje en educación y política. Chile: Dolmen.

Maturana, H., \& Varela, F. (1999). El árbol del conocimiento. Las bases biológicas del entendimiento humano. Chile: Universitarias.

Martínez, M. (2009). Nuevos Paradigmas en la investigación. Caracas: Alfa.

Martínez, M. (2008). Práctica y teoría metodológica en las ciencias sociales y humanas hoy. Foro presentado en las IV Jornadas de Innovación e Investigación Educativas REDINE. Universidad Centroccidental Lisandro Alvarado Barquisimeto, Venezuela.

Moreno, A. (1995). El aro y la trama. Episteme, modernidad y pueblo. Caracas: Centro de investigaciones populares.

Prigogine Ilya et Stengers, I. (1986). La Nouvelle Alliance. Paris: Gallimard.

Rivera, O. (2000). La gestión del conocimiento en el mundo académico: ¿Cómo es la universidad de la era del conocimiento? España: Facultad de CCEE y Empresariales, Universidad de Deusto.

Parra, M. (2008). Las intimidades de la academia. Un estudio cuanti-cualitativo sobre la dinámica de la profesión académica. Maracaibo: Universidad del Zulia.

Sander, B. (1996) Gestión educativa en América Latina. Construcción y reconstrucción del conocimiento. Argentina: Troquel.

Vieira, E. (2011) Contruir conceitos que muden de fato o mindset exige território mental sustentável e democrático. Recuperado el 06 de agosto de 2012 de http://www. evandrovieiraouriques.com

Vieira, E (2009). Território Mental, o nó górdio da democracia. Democracia viva, 42, pp. 76-81. 